\title{
PUITS ET FOSSES DE LA FONTAINE DES MERSANS À ARGENTOMAGUS. DÉPOTOIRS OU DÉPÔTS VOTIFS?
}

\author{
par Jacques ALLAIN, Isabelle FAUDUET et Jacques DUPOUX $\dagger$
}

\section{DESCRIPTION ET INTERPRÉTATION}

Au cours des fouilles menées par l'un de nous (J.A.) depuis vingt ans à Argentomagus, SaintMarcel (Indre), une importante série de puits et de fosses a pu être explorée sur cet ancien oppidum secondairement romanisé. Outre les nombreuses cavités déjà explorées au gré des circonstances sur l'ensemble de l'habitat, trente-sept ont été fouillées à l'intérieur d'une aire cultuelle comprenant deux temples de type indigène et leurs annexes ${ }^{1}$.

Non loin de là, quatorze autres ensembles clos analogues ont été découverts à l'emplacement et aux abords immédiats d'un important Monument des Eaux, communément appelé Fontaine des Mersans ${ }^{2}$ (fig. 1).

Cet édifice long de $25 \mathrm{~m}$, large de $12 \mathrm{~m}$ est implanté, comme l'aire cultuelle évoquée ci-dessus entre deux voies e.-o. parallèles. Il se compose d'un bassin central de $6 \mathrm{~m}$ de côté aménagé au fond d'une vaste excavation, à $3 \mathrm{~m}$ au-dessous du niveau des voies. Ce bassin était à l'origine parfaitement étanche. Un revêtement de grandes dalles calcaires liées entre elles par des joints d'étanchéité venait doubler, au fond, un lit de béton rose posé lui-même sur une semelle d'argile rapportée.

1 J. Allain, L. Fleuriot et L. Chaix, Le Vergobret des Bituriges à Argentomagus, essai d'interprétation d'une fosse cultuelle, Revue Archéologique de l'Est et du Centre-Est, XXXIII, 1981, p. 11-32.

2 Ce monument est figuré dans G.-Сh. Picard, Les sanctuaires d'Argentomagus, Comptes rendus de l'Académie des Inscriptions et Belles-Lettres, 1971, p. 621.
Quatre piliers d'angle renforçaient l'ensemble et supportaient probablement la vaste charpente d'un impluvium. Celui-ci recouvrait les deux larges escaliers n. (14 marches) et s. (15 marches) qui, partant des deux voies, descendent au bassin. Deux murs parallèles, en petit appareil à l'e., en grand appareil à l'o., enserrent le monument. A leur pied, s'allongent trois rangées de gradins bas.

Le bassin était alimenté, outre les caux de pluies, par une canalisation en bois à frettes de fer raccordée au réseau urbain obliquement aménagée sous l'escalier $n$. et qui se déversait par deux griffons aujourd'hui disparus. L'eau s'évacuait par un tropplein dans une canalisation souterraine voûtée, haute de $2 \mathrm{~m}$, large de $0,70 \mathrm{~m}$, ouverte dans le mur e. du monument.

L'originalité de ce monument est telle qu'on ne connaît pas en Gaule de véritables comparaisons; mais plusieurs inscriptions votives sur les piliers d'angle permettent de lui attribuer avec vraisemblance une fonction à la fois municipale et religieuse.

Dès son dégagement, nous avons été frappés par l'aspect chaotique de l'escalier s. En sept points, les marches étaient disloquées ou effondrées. La fouille, dont le plan d'ensemble est représenté sur la figure 2, allait montrer qu'à chacun de ces accidents correspondaient une fosse ou un puits ${ }^{3}$ :

- le long de la marche la plus élevée, une cavité importante a été reconnue et partiellement fouillée (14);

3 Le premier puils expluré a élé publié : R. Alber't, Un ensemble céramique clos avec vase Drag. 11 à Argentomaqus (Indre), Revue Archéologique du Centre, 1973, p. 95. 

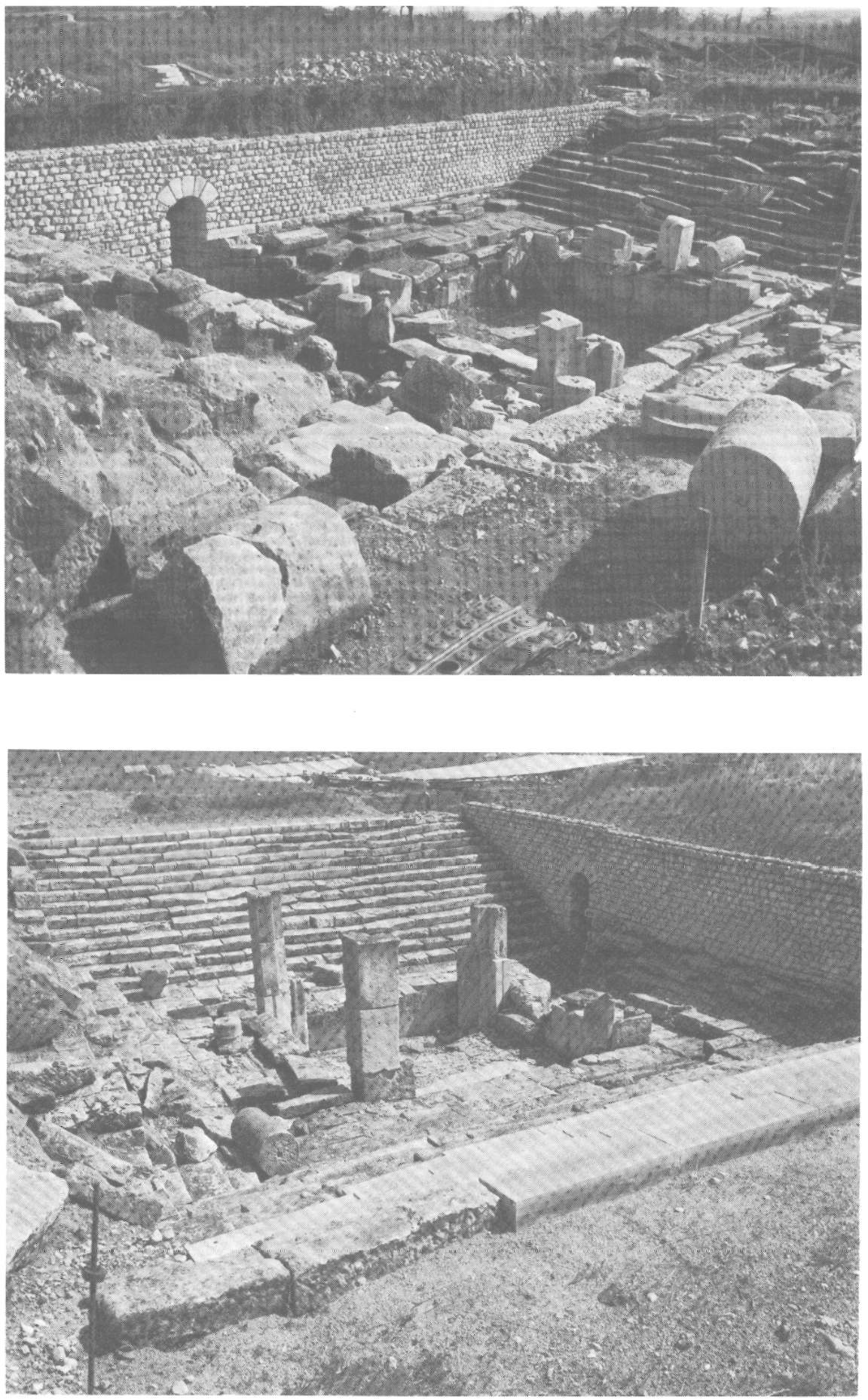

Fig. 1 - La Fontaine des Mersans. En haut, vue générale prise du n. Seul le mur e. a été restauré. Au premier plan, vestiges de la tranchée de canalisation afférente. Trois des marches de l'escalier $n$. utilisées pour exhausser tardivement le pourtour du bassin sont toujours en place. L'escalier s. conserve son aspect disloqué du fait du tassement des fosses et puits sous-jacents. En bas, vue générale prise du s. après restauration de l'escalier $\mathbf{n}$. Trois marches ont été laissées en témoins sur le bord o. du bassin. De même, dans l'angle n.-e. de cet escalier, on distingue un léger tassement attestant la présence d'une fosse sousjacente. Les piliers d'anglc ont ćté remis en place. 


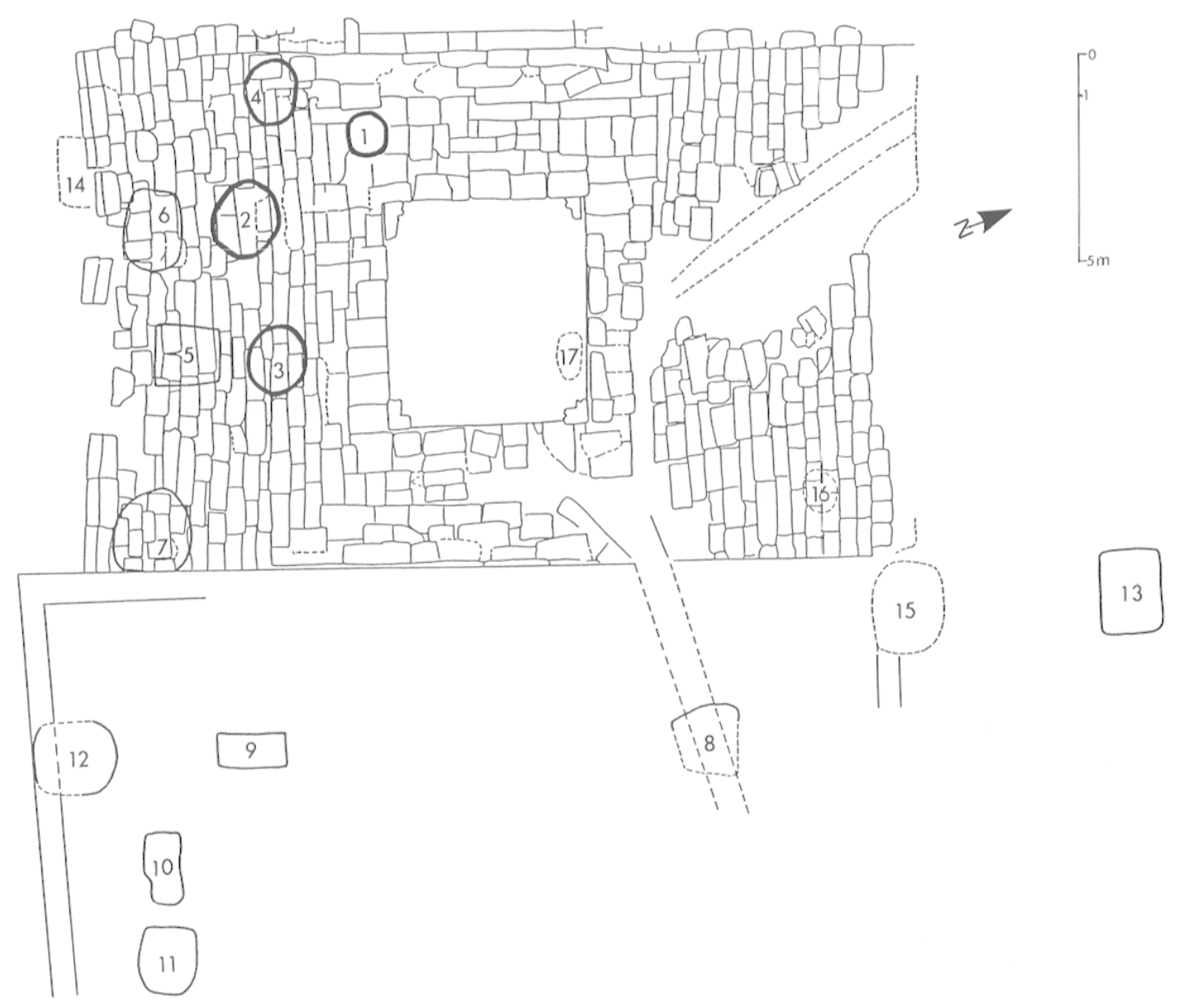

Fig. 2 - Plan d'ensemble. Deux larges escaliers n. et s. conduisent au bassin de la fontaine (au centre) et ses deux canalisations. Le monument recouvre les puits (nos 1 à 4) et les fosses (nos 5 à 17).

- deux autres cavités non fouillées sont probablement incluses dans le périmètre du monument : l'une se traduit par un infléchissement de l'escalier $\mathrm{n}$. à peu de distance du mur e. (16). L'autre (17) se situerait sous le bassin lui-même, là où se déversaient les griffons. C'est du moins l'explication la plus probable d'un tassement localisé en ce point;

- à proximité immédiate de l'angle s.-e. du monument, mais au-delà du mur en petit appareil, trois fosses ont été complètement fouillées $(9$, $10,11)$. Une quatrième n'a été que partiellement explorée (12);

- une fosse, parallélépipédique, appareillée en pierres sèches, était surcroisée obliquement par la canalisation émissaire à $3,50 \mathrm{~m}$ de son porche d'accès (8).

A l'angle n.-e., tangentiellement au mur e., une cavité considérable a été reconnue mais son exploration posait de si graves problèmes de sécurité que sa prospection a dû être abandonnée (15). Sa forme et ses dimensions exactes nous sont inconnues. Enfin, au $\mathrm{n}$. de la fontaine, une autre fosse rectangulaire a été reconnue et fouillée sous la voie elle-même (13).

Comme dans l'aire cultuelle, il est possible de distinguer puits et fosses (voir tableau page 108).

Les puits $(1,2,3,4)$ ont une ouverture étroite ( $1 \mathrm{~m}$ à $1,20 \mathrm{~m}$, une forme cylindrique et une profondeur d'au moins quatre fois leur diamètre. Les quatre puits découverts sous l'escalier $\mathrm{s}$. atteignent une profondeur de $4,20 \mathrm{~m}$ à $4,75 \mathrm{~m}$. Deux se rétrécissent à leur base sensiblement dans leur dernier demi-mètre.

Après le limon superficiel, ils traversent une couche de galets quartzeux et $3 \mathrm{~m}$ de sable rouge compact du quaternaire ancien. Certains atteignent la couche d'argile éocène jaune qui nappe le socle calcaire de l'oppidum, deux s'arrêtent à une cinquantaine de centimètres au-dessus de l'argile. Aucune trace certaine de cuvelage n'a été retrouvée; la mise en place ultérieure des escaliers ne permet pas de savoir s'il y a eu ou non des margelles.

On peut se demander si ces puits ont eu un rôle 
TABLEAU

Ensembles clos de la Fontaine des Mersans : tableau synoptique des éléments significatifs.

\begin{tabular}{|c|c|c|c|c|c|c|c|}
\hline & \begin{tabular}{|c|} 
Profondeur \\
maxima
\end{tabular} & Forme & $\begin{array}{l}\text { Vestiges } \\
\text { osseux }\end{array}$ & Sigillée & $\begin{array}{c}\text { Autres } \\
\text { Céramiques* }\end{array}$ & Monnaies & Divers \\
\hline \multicolumn{8}{|l|}{ Puits } \\
\hline 1 & $4,74 \mathrm{~m}$ & cylindrique & $\begin{array}{l}\text { oiseaux } \\
\text { (fragments) } \\
\text { os sciés }\end{array}$ & $\begin{array}{l}\text { Drag. } 11,24 / 25 \\
\text { Drag. } 17,29 \\
\text { Ritt. } 12\end{array}$ & $\begin{array}{l}\text { cruche blanche } \\
\text { céramique d'Aco }\end{array}$ & $\begin{array}{l}\text { Denier de la } \\
\text { République } \\
2 \text { potins }\end{array}$ & \\
\hline 2 & $4,60 \mathrm{~m}$ & cylindrique & bois de cerf & $\begin{array}{l}\text { Drag. } 16 \\
\text { marque } \\
\text { ATEIUS }\end{array}$ & $\begin{array}{l}\text { lot cruches } \\
\text { blanches }\end{array}$ & & $\begin{array}{l}\text { rondelles terre } \\
\text { cuite, lampe } \\
\text { (fragments) } \\
\text { creuset }\end{array}$ \\
\hline 3 & $4,20 \mathrm{~m}$ & cylindrique & & Drag. 27,29 & & $\begin{array}{l}\text { as autel de Lyon } \\
\text { as } \mathrm{I}^{\mathrm{er}} \mathrm{s} \text {. }\end{array}$ & lot de pesons \\
\hline 4 & $4,40 \mathrm{~m}$ & cylindrique & crâne de bouc & $\begin{array}{l}\text { Drag. } 16,26 \\
\text { Ritt. } 5\end{array}$ & $\begin{array}{l}\text { ovoïde à fond } \\
\text { découpé }\end{array}$ & $1 / 2$ as de Nîmes & $\begin{array}{l}\text { lot de pesons, } \\
\text { creusets }\end{array}$ \\
\hline $\begin{array}{r}\text { FOSSES } \\
5\end{array}$ & $2,35 \mathrm{~m}$ & $\begin{array}{l}\text { parallélépi- } \\
\text { pédique }\end{array}$ & & Drag. 16 & $\begin{array}{l}\text { assiettes dont } \\
\text { une intacte }\end{array}$ & $\begin{array}{l}\text { hémi-obole } \\
\text { Massalia - } \\
4 \text { gauloises }\end{array}$ & $\begin{array}{l}\text { creusets } \\
\text { outils de fer }\end{array}$ \\
\hline 6 & $?$ & ovalaire & & $\begin{array}{l}\text { fragm. arrétine } \\
\text { TETIUS- } \\
\text { SAMIA }\end{array}$ & assiettes, divers & $\begin{array}{l}\text { as de Nîmes } \\
3 \text { gauloises }\end{array}$ & $\begin{array}{l}\text { manche de patère } \\
\text { en terre cuite }\end{array}$ \\
\hline 7 & $2,50 \mathrm{~m}$ & cubique & $\begin{array}{l}\text { rachis de bovidé } \\
\text { bois de cerf }\end{array}$ & $\begin{array}{l}\text { Drag. } 16 \\
\text { fragm. } \\
\text { Drag. } 15,17\end{array}$ & $\begin{array}{l}\text { cruche avec } \\
\text { dédicace, jarre }\end{array}$ & & $\begin{array}{l}\text { lampe } \\
\text { (fragments) }\end{array}$ \\
\hline 8 & & $\begin{array}{l}\text { parallélépi- } \\
\text { pédique }\end{array}$ & bois de cerf & $\begin{array}{l}\text { fragm. } \\
\text { Drag. } 17,29\end{array}$ & cruche blanche & bronze de Claude & $\begin{array}{l}\text { armes miniatures } \\
\text { lamelle en plomb }\end{array}$ \\
\hline 9 & $3,65 \mathrm{~m}$ & id. & $\begin{array}{l}3 \text { rachis de bovidé } \\
\text { bois de cerf }\end{array}$ & & & 1 gauloise & $\begin{array}{l}\text { pigments dans } \\
\text { des vases }\end{array}$ \\
\hline 10 & $2,80 \mathrm{~m}$ & id. & $\begin{array}{l}\text { bovidés, suidés } \\
\text { (nombreux } \\
\text { fragments) }\end{array}$ & Drag. 16 & cruche blanche & & \\
\hline 11 & $3,20 \mathrm{~m}$ & $\begin{array}{l}\text { hémisphéri- } \\
\text { que }\end{array}$ & $\begin{array}{l}\text { bois de cerf } \\
\text { bovidés, suidés } \\
\text { (nombreux } \\
\text { fragments) }\end{array}$ & $\begin{array}{l}\text { Drag. 16, 17, } \\
24 / 2523\end{array}$ & jarre & & \\
\hline 12 & $2,50 \mathrm{~m}$ & $\begin{array}{l}\text { hémisphéri- } \\
\text { que }\end{array}$ & $\begin{array}{l}\text { crâne et bois de } \\
\text { cerf, crânes de } \\
\text { bovidé et chien }\end{array}$ & $\begin{array}{l}\text { fragm. } \\
\text { RASINUS }\end{array}$ & fragments & 2 gauloises & $\begin{array}{l}\text { lampe } \\
\text { (fragments) }\end{array}$ \\
\hline 13 & $2,50 \mathrm{~m}$ & $\begin{array}{l}\text { parallélépi- } \\
\text { pédique }\end{array}$ & $\begin{array}{l}\text { bois de cerf } \\
\text { os sciés }\end{array}$ & $\begin{array}{l}\text { fragm. Drag. } 16 \\
\text { HERTORIVS }\end{array}$ & $\begin{array}{l}\text { amphores ita- } \\
\text { liques, cérami- } \\
\text { que d'Aco }\end{array}$ & $\begin{array}{l}\text { as de Nîmes } \\
6 \text { gauloises dont } \\
3 \text { Atectori }\end{array}$ & $\begin{array}{l}\text { lampe } \\
\text { (fragments) } \\
\text { peson }\end{array}$ \\
\hline 14 & & $i d$ & rachis de bovidé & $\begin{array}{l}\text { Drag } 11 \text { et } 16,17 \\
\text { (fragm.) } \\
\text { TETIVS } \\
\text { SAMIA }\end{array}$ & jarre fragmentée & 2 bituriges & $\begin{array}{l}\text { lampe } \\
\text { (fragments) } \\
\text { creusets, moules }\end{array}$ \\
\hline
\end{tabular}

* Les cruches blanches, plus ou moins abondantes et fragmentées sont difficiles à dénombrer. Leur pied est dégagé mais le modèle à pied balustre est absent. 
fonctionnel d'approvisionnement en eau. Au fond des puits, la texture du sable rouge se modifie dans ses cinquante derniers centimètres par perte de ses éléments fins et donc de sa compacité; on observe des ferritisations secondaires probablement amorcées par des éléments indéterminables. Cela laisse à penser qu'il y avait au contact de l'argile une faible nappe aquifère, aujourd'hui disparue. En tout cas, jusqu'ici, il n'est connu sur le plateau des Mersans aucune source pérenne susceptible d'alimenter l'oppidum.

Ces quatre puits ont un remplissage archéologique analogue qui s'est effectué en plusieurs temps : tout au fond, une couche noirâtre, riche en matières organiques et en mobilier archéologique, est recouverte d'un bouchon argilo-sableux stérile; un nouveau dépôt, rappelant celui du fond, est à son tour occulté par un niveau stérile avec parfois (2), une deuxième alternance du même type. Pour un même puits aucune différence chronologique appréciable n'est discernable dans le mobilier livré par les différentes strates. Au contraire, dans un cas, il y a coaptation entre un tesson du niveau supérieur et les débris d'un vase du niveau inférieur. Le bon état relatif des parois pourtant fragiles, incline à penser que les étapes du comblement se sont succédées dans un laps de temps très court. Le volume du remplissage secondairement infiltré qui a infléchi, voire englouti les marches, atteint ou dépasse le tiers de la capacité globale de la cavité. Il n'y a donc pas eu tassement intentionnel du remplissage primitif; au contraire, le volume initial des matières organiques lentement décomposées et accompagnant le mobilier archéologique devait être assez considérable. La fouille de ces puits, rendue dangereuse par la nature sableuse du terrain a nécessité l'emploi de cuvelages amovibles superposés.

Les fosses se différencient des puits tout d'abord par leurs dimensions. En général plus vastes en gueule (environ $2 \mathrm{~m}^{2}$ ), elles sont sensiblement moins profondes; les unes sont plus ou moins hémisphériques $(6,11,12)$, les autres sont parallélépipédiques, très régulièrement et soigneusement creusées $(5,7$ à 10) (fig. 3). Leur profondeur atteint ou dépasse même leur plus grande largeur au sommet. Le remplissage de la plus profonde (9) s'est, comme pour les puits, effectué en trois temps, avec bouchon

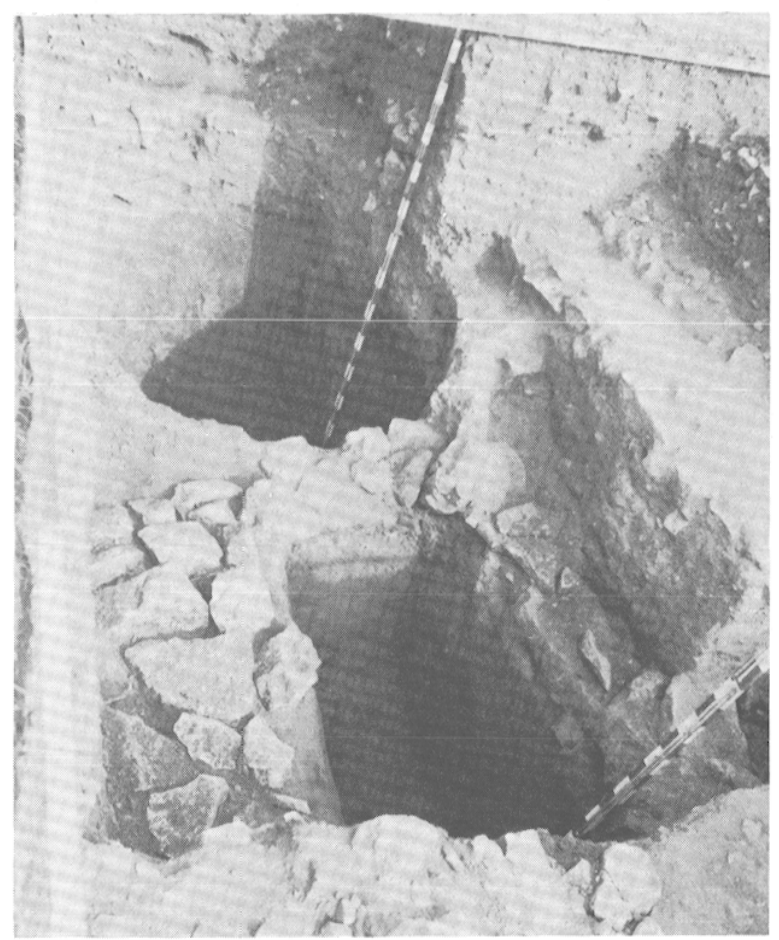

Fig. 3 - Fosses 10 et 11 situées aux abords s.-e. de la fontaine.

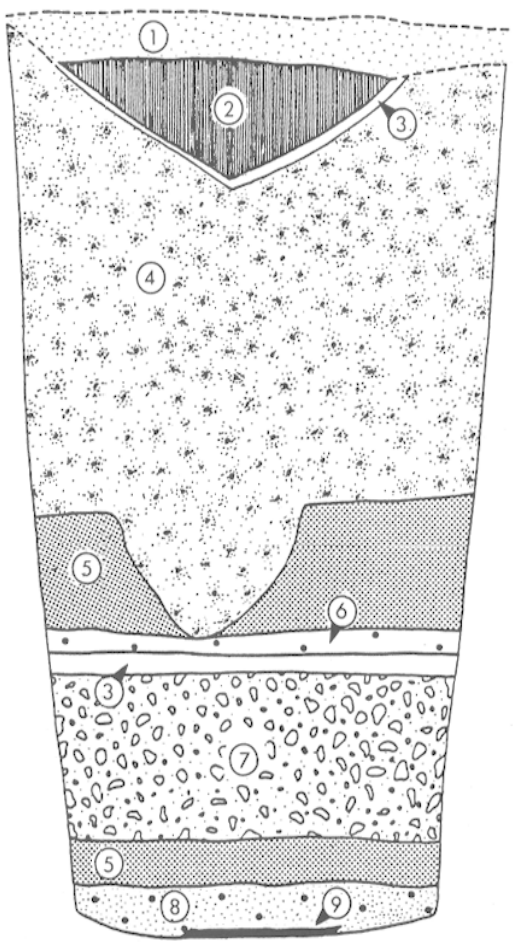

Fig. 4 - Coupe de la fosse 5 . - 1, sable; 2 , terre noire; 3 , sable rouge stérile; 4 , couche noire cendreuse; 5 , sable jaune; 6 , cendre et boue; 7 , sable, boue et argile; 8 , sable, boue et cendre; 9 , fer (échelle : $1 / 25^{\mathrm{e}}$ ). 


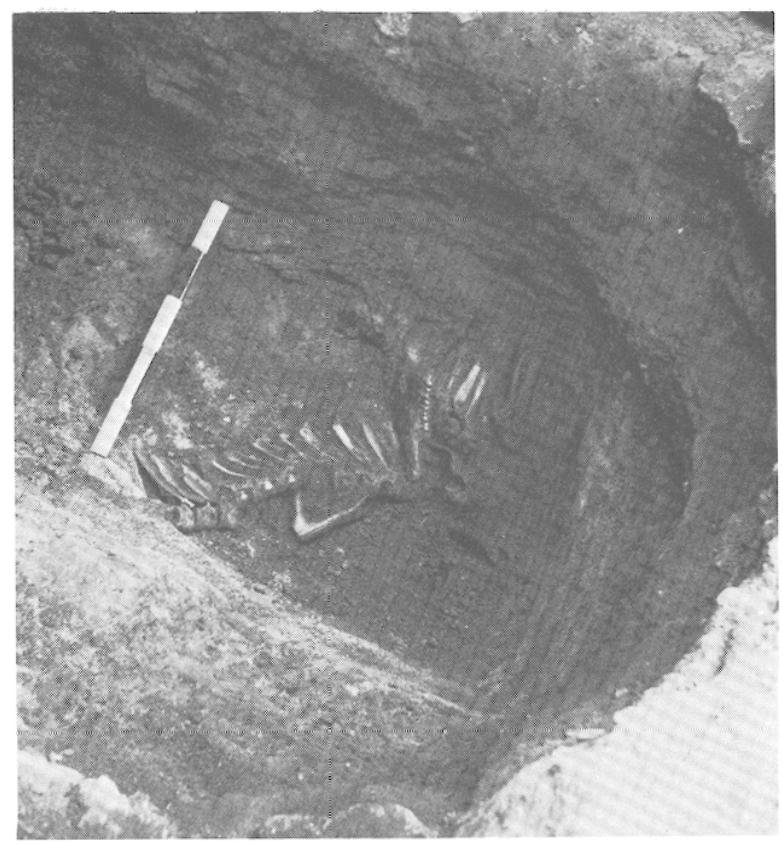

Fig. 5- Fosse 9. Rachis de bovidé et fragment de bois de cerf.

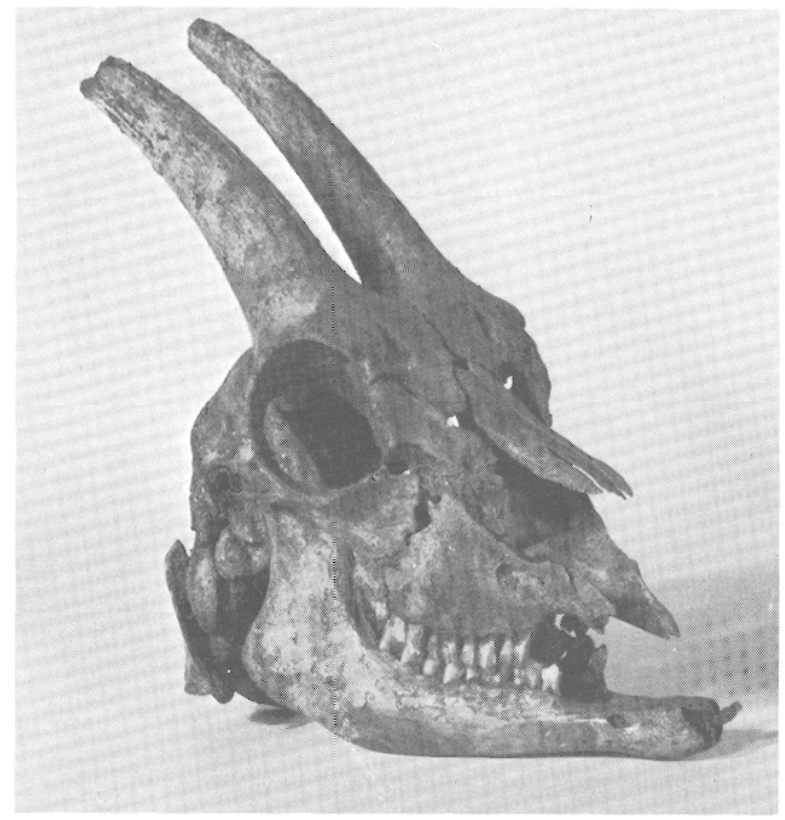

Fig. 6 - Puits 4. Crâne de bouc.

stérile interposé ${ }^{4}$. Il semble que la fosse 5 , à section rectangulaire également, ait eu un remplissage analogue (fig. 4). Le plus souvent, pourtant, celui-ci,

4 Un compte-rendu des fouilles menées sur les abords sud-est de la Fontaine est publié : I. Fauduet, La Fontaine des Mersans à Argentomagus (Indre), les débuts de l'occupation du site d'après les dernières découvertes, Actes $d u 44$ Congrès de la Fédérulion des Societés Savantes du Centre de la France, Châteauroux, mai 1984 (1985), p. 24. après dépôt d'un premier lit à haute densité organique est réalisé par une série de déversements secondaires variables par leur nombre, leur importance et leur nature.

Il est concevable dès lors que ces fosses, malgré un emplacement parfois incompatible avec la notion de rejets domestiques, aient pu être qualifiées de dépotoirs. L'analyse plus fine et détaillée des remplissages a compliqué singulièrement la question.

En effet, la relative abondance d'ossements déconnectés évoque des déchets culinaires. Ainsi, la présence isolée d'un quartier animal en connexion anatomique pourrait être considérée comme fortuite. Mais ce dépôt est répétitif : deux rachis de veau avec leurs côtes et quatre mandibules au fond de la fosse 9 (fig. 5) puis dans la deuxième couche fertile, une autre pièce analogue qu'accompagne un fragment de bois de cerf et enfin dans la couche profonde du puits 4 , un crâne de bouc (fig. 6). Le caractère cultuel apparaît donc probable ${ }^{5}$. La présence d'autres fragments de bois de cerf plus ou moins travaillés fait penser à des déchets de tabletterie. Lorsque s'y ajoute un calvarium complet de cerf après sciage des bois, cette première interprétation paraît insuffisante.

L'examen des éléments céramiques conduit à des conclusions similaires. Il est certes d'observation courante qu'un vase brisé à l'usage soit jeté en bloc à la décharge et que ses tessons se retrouvent presque en contiguïté. La découverte au fond du puits 1 de plusieurs vases totalement différents par leur qualité et leur destination, tous inaptes au puisage, rend caduque l'hypothèse du rejet domestique.

Plus compatible à première vue avec une destination fonctionnelle, serait la présence au fond d'un autre puits (2), d'une série de cruches blanches, bien que leur utilisation pour puiser de l'eau soit fonctionnellement bien surprenante. Plusieurs cruches identiques se trouvaient au fond d'une fosse et non plus d'un puits : cela requiert une autre explication. Quand le bris de la cruche a été précédé par la cassure intentionnelle du pied, dans le but évident de lui retirer toute valeur fonctionnelle (fig 7) - il en existe plusieurs exemples - une autre motivation doit être recherchée.

La présence d'une cruche du même type, intégralement reconstituée, portant en haut de sa panse une inscription dédicatoire ${ }^{6}$ (fig. 10c) fait

5 Cf. supra, p. 105, note 3.

6 Cf. infra, p. 113, note épigraphique, par J. Dupoux. 


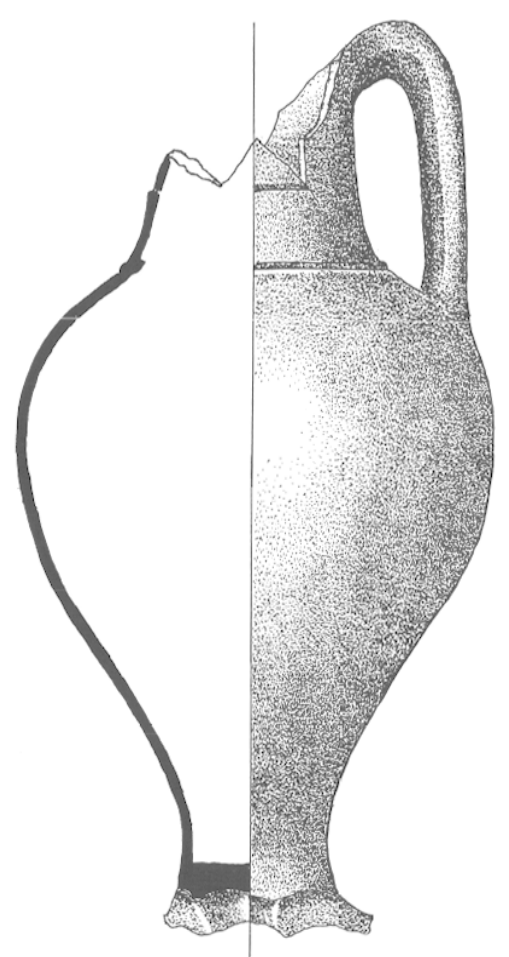

Fig. 7 - Fosse 10. Cruche blanche. Noter la cassure intentionnelle du pied (hauteur : $26 \mathrm{~cm}$ ).

perdre à la fonction "dépotoir» toute vraisemblance. Offrandes, dépôts votifs, sacrifices, nous ne choisirons pas entre ces différents termes, faute d'informations suffisamment précises, tout en reconnaissant à ces ensembles clos un caractère rituel.

Cette interprétation se trouve renforcée par la nature de certains objets. Un manche de patère en terre cuite terminé par une tête de bélier, semblable à ceux des dépôts funéraires de Rhénanie, découvert dans la fosse 6 conforte le rôle que joue la patère dans le rituel sacrificiel.

Les découvertes numismatiques y sont assez courantes. Ainsi, la fosse 13 contenait six monnaies gauloises et un as de Nîmes. Le pouvoir libératoire de ces petites espèces de bronze était pourtant assez faible. En revanche, un denier républicain de la gens Scribonia (fig. 8) associè à deux ou trois petits bronzes gaulois, corrodés à l'extrême, se trouvait dans la partie supérieure du remplissage intentionnel du puits 1 . Au revers, figure le puits sacré du forum avec la légende puteal. Il est permis de voir dans la présence de ce denier plus qu'un abandon fortuit. D'autre part, la fosse 8 contenait les miniatures de deux armes votives : un glaive muni de son fourreau (fig. 9) et un bouclier indigène (long. : $15 \mathrm{~cm})^{7}$. Ces

7 I. Fauduet, Miniature ex-voto from Argentomagus (Indre), Britannia, XIV, 1983, p. 97.

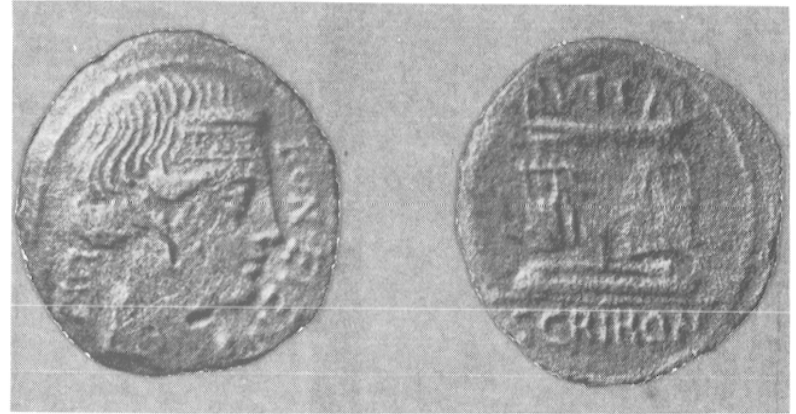

Fig. 8 - Puits 1. Partie supérieure du remplissage. Denier de la République : à l'avers, tête de Bonus Eventus; au revers, légende PUTEAL et SCRIBON(ius); au centre, le puits du forum (diamètre : $18 \mathrm{~mm}$ ).

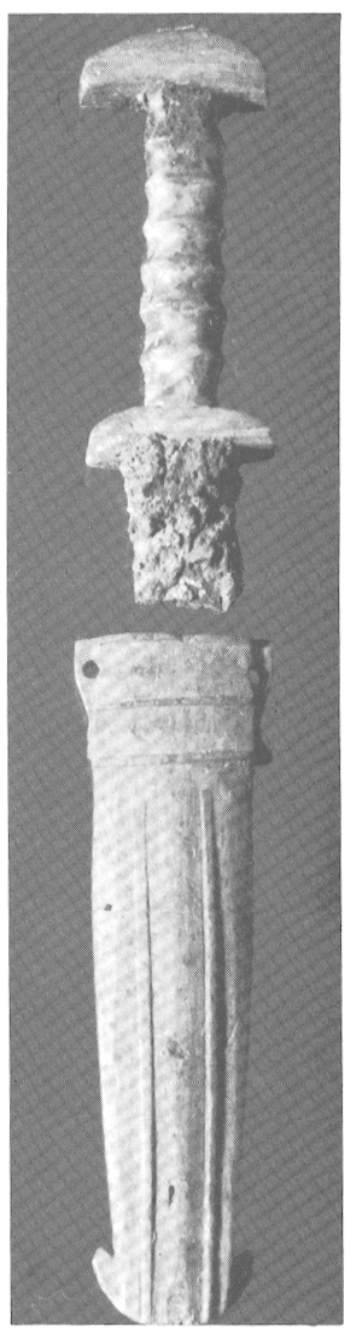

Fig. $9-$ Fosse 8. Glaive miniature et son fourreau (longueur du glaive : $12,6 \mathrm{~cm}$ ).

ex-voto étaient accompagnés d'une fibule, d'un moyen bronze de Claude, d'une cruche blanche et de fragments de bois de cerf. De fait, lc caractère cultuel de ces dépôts semble manifeste. 
Tous ces exemples proviennent d'ensembles clos apparemment antérieurs à l'édification du monument. La fosse 13, rectangulaire a été creusée dans la grande voie e.-o. qui longe l'escalier $n$. de la Fontaine. La coupe de la voie a permis de dater avec précision l'assise la plus ancienne : épais blocage lié au mortier de chaux recouvert d'un lit cendreux bien situé aux environs de 30 av. J.-C. Et pourtant, la fosse 13, datable par son remplissage de la fin du règne d'Auguste, a été creusée à travers cet ouvrage d'art et entame l'agglomérat de sable rouge en place. Ce sable extrait et les éléments arrachés au blocage ont été remis pêle-mêle sur le dépôt organique et cendreux qui garnit le fond. L'effet de tassement s'est fait sentir pendant des siècles à travers les recharges successives jusqu'au dernier état de la voie. Il fallait un motif grave et tout à fait étranger à la notion d'urbanisme pour perturber ainsi, volontairement, un axe routier majeur en pleine ville.

De l'important mobilier extrait de ces puits et fosses, quelques exemples nous autorisent à conclure au caractère rituel de leur remplissage, même s'il subsiste un doute sur le rôle initialement fonctionnel des puits. Ceci permet aussi d'en préciser la chronologie.

Ccs divers ensembles clos ne sont pas contemporains. Leur remplissage s'échelonne du tout début du $\mathrm{I}^{\mathrm{er}} \mathrm{s}$. à l'époque de Néron - Vespasien. Il n'y a donc pas de rapport direct entre l'érection du monument et la mise en place des dépôts. Cet étalement dans le temps laisse supposer que, dès avant l'édification du monument, son emplacement avait un caractère sacré : la présence d'ex-voto dans la fosse claudienne (8) sous la canalisation émissaire est particulièrcment éloquente. La disposition orthogonale des fosses extérieures par rapport aux axes du monument tend à confirmer cette continuité. Bien que, à quelques mètres à l'o., nous ayons observé les vestiges indubitables d'une occupation antérieure à la Conquête, le périmètre immédiat de la Fontaine des Mersans n'a jusqu'ici rien livré qui puisse remonter à la Gaule Indépendante.

Le plus récent des ensembles clos sous-jacents au monument fournit un précieux terminus a quo. La Fontaine des Mersans s'inscrit dans le programme de romanisation générale des structures urbaines d' $A r$ gentomagus durant le dernier quart du $\mathrm{I}^{\mathrm{er}} \mathrm{s}$. de notre ère.

Le caractère rituel du remplissage de la majorité de ces quatorze puits et fosses parait vraisemblable. Cependant, cet aperçu devra être notablement complété et affiné. Une comparaison entre les ensembles clos ci-dessus décrits et ceux qui ont été fouillés dans le périmètre des deux fana et les structures analogues issues des zones d'habitat permettra de mieux apprécier similitudes et différences et d'éclairer des aspects encore mal connus des cultes indigènes et de leurs survivances.

Jacques Allain et Isabelle Fauduet 
Le vase portant cette inscription (fig. 10) se trouvait intentionnellement brisé dans la fosse 7 de la fontaine avec plusieurs autres pièces de céramique, l'ensemble paraissant datable de l'époque de Tibère (14-37).

Cette inscription est constituee par dix-sept lettres. C'est le plus long des graffites découverts jusqu'à présent dans les fouilles d'Argentomagus :

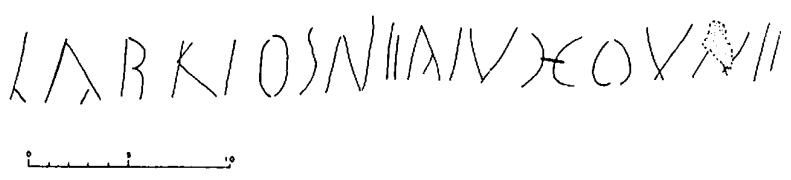

La gravure des lettres est parfois imparfaite. Certains traits se croisent ou ne se joignent pas, d'autres sont légérement tremblés et sinueux (S).

La quatrième lettre $\mathrm{K}$ est une forme connue de $\mathrm{R}$ (graffites et marques de potieri, citee par Holder ${ }^{8}$. Les // sont généralement considérés comme des $\mathrm{E}$. Le - ne semble pas pouvoir être interprété autrement que par X: la ligature horizontale est moins profonde et plus large que les autres traits de l'inscription, comme si le style du graveur, peut-être plus oblique, avait moins bien pénétré dans l'engobe. La seizième lettre est incomplète (il manque un éclat) mais paraît bien devoir être considérée comme un $\mathrm{N}$.

Nous lisons donc : LABRIOS NEAI UXOVNE.

Avant d'aller plus avant dans l'interprétation de cette inscription, rappelons brièvement le contexte archéologique des découvertes.

Le puits 1, dont l'utilisation pour l'approvisionnement en eau n'est pas attestée, a été comblé pendant le troisième quart du $\mathrm{i}^{\mathrm{er}} \mathrm{s}$.

La partie supérieure du remplissage contenait un denier de Paulus Emilius Lepidus et de L. Scribonius Libo (fig. 7), émis vers 55 av. J.-C. et dont le revers représente le puits scribonien ${ }^{9}$.

8 A. Holder, Altceltischer Sprachschatz, Leipzig, I, 1896 ; II, 1904 ; III, 1907; réimpression : Graz, 1961-1962.

9 En réalité, un puteal, situé sur le forum, destiné à commémorer la chute de la foudre en ce point.
La présence dans le comblement intentionnel d'un puits, d'un denier vieux de plus d'un siècle, représentant lui-même un puits ne peut être le fait du hasard. Le contenu de ce comblement pourrait être interprété comme des excuses auprès de la divinité de ce puits lors de la constrution de la fontaine monumentale.

La correspondance entre la signification de certains dépôts et de leur lieu d'enfouissement nous guide pour interpréter l'inscription.

LABRIOS a l'allure d'un andronyme au nominatif, formé avec contraction, sur labar (éloquent) ${ }^{10}$.

NEAI paraît être un datif singulier, de même que Labrios est contracté, NEAI ne serait-il pas une contraction de NEVIAI (nouvelle) ${ }^{11}$ ?

VXOVNE : le mot ne semble attesté nulle part, mais il serait formé de $V X$ (haut ou extrême) et de GVNO (élevé).Il est donc possible d'envisager l'interprétation suivante : LABRIOS NEAI VXOVNE: Labrios à la nouvelle très haute (j'ai dédié).

Nous pouvons alors envisager l'hypothèse suivante : peu de temps avant le comblement du puits 1 et le sacrifice expiatoire à son ancienne divinité, la fosse 7 contenant des offrandes à la nouvelle divinité a été creusée. Cette hypothèse est effectivement fort risquée mais elle peut s'insérer dans l'ensemble des témoignages archéologiques.

M. Lejeune, consulté par lettre, émet un certain nombre de réserves:

1 - il s'étonne de la présence d'une fin de mot en $-a i$, cette diphtongue finale s'étant réduite à $-\imath$ en gaulois dès avant l'ère chrétienne, et il se demande s'il n'y a pas là une faute de gravure (omission de la barrette d'un $\mathrm{t}$ ) : on aurait alors ici un verbe (présent $3^{e}$ singulier de première conjugaison : -at) quelque explication qu'on en cherche (par exemple, à partir de "nei-, "faire descendre", "plonger"?);

2 - le second mot ne peut rien avoir à faire avec un adjectif "nouveau", dont le thème est novio- - en gaulois -;

10 Sont cités par A. Holder, op. cit., Labaros, Lambreus (forme contractée, nasalisée et latinisée).

11 La forme NERVIOS à côté de Novios est attestée par G. Dotrin, La langue gauloise, Paris, 1920. 

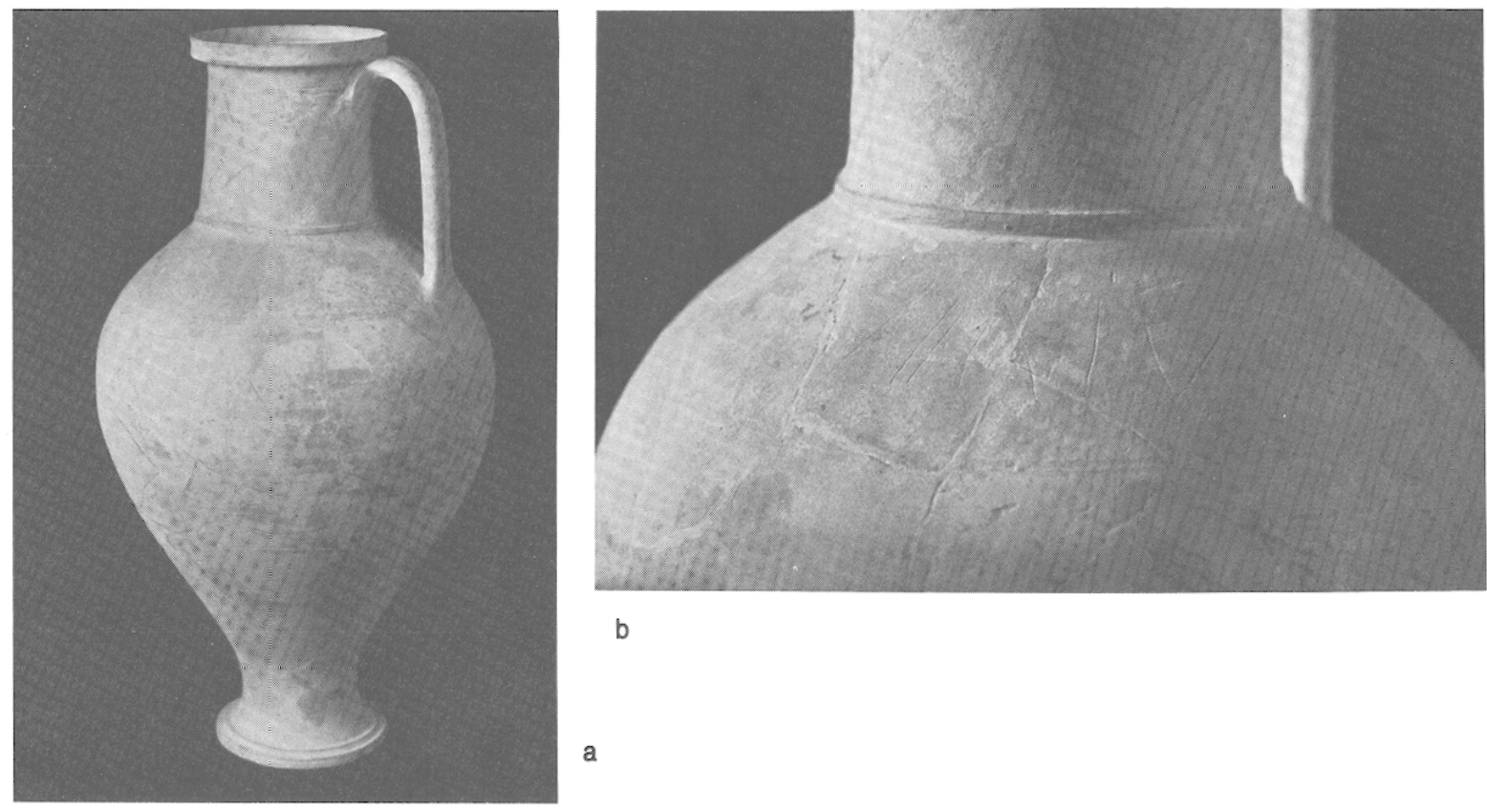

b

a

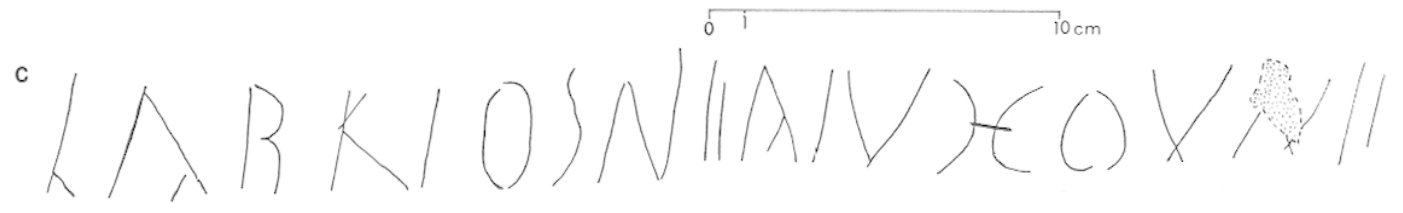

Fig. 10 - Fosse 7. a, cruche blanche portant un graffite sur l'épaule (hauteur : $33 \mathrm{~cm}$; diamètre : $21 \mathrm{~cm}$ ). b, détail de l'épaule de la cruche et du graffite. c, relevé du graffite.

3 - si la double haste finale du dernier mot entendait être une notation de $\bar{i}$, nous pourrions avoir là un datif singulier de première déclinaison, nom de la divinité dédicataire: en l'espèce, une déesse Uxouna (dont le nom évoque celui du dieu Uxovinos, CIL, XII, 1062).

Le texte suggéré par M. Lejeune paraît obscur, NEAT NIAT ou NIYAT n'étant pas attestés.

En outre, nous remarquons que NEVIOS NEVIA est signalé par G. Dottin ${ }^{12}$ : un datif en -AI est certes un archaïsme. Toutefois, la survivance, au début de notre ère, de cette forme dans la langue peut s'accorder avec le conservatisme des Bituriges : nous savons qu'un vergobret venait encore sacrifier à Argentomagus vers $20-30^{13}$.

Enfin, quelques découvertes faites dans le Vaucluse peuvent compléter l'interprétation du nom UXOVNE. Une dédicace au dieu UXOVNE provient de Bonnieux, à la frontière $\mathrm{n}$. du territoire du

12 Cf. supra, p. 113, note 11.

13 Cf. supra, p. 105, note 1. peuple ligure des Dexiviates ${ }^{14}$ à quelques kilomètres de leur capitale (Castellar-de-Cadenet) où ont été trouvées deux dédicaces à la déesse DEXIVA ou DEXSIVA (GIL, XII, 1064), une autre provenant du Pertuis (CIL, XII, 1062). Les peuples ligures ont été tardivement celtisés : il pourrait donc ne pas sembler évident qu'une déesse VXOVINA soit signalée au cœur du territoire celtique, alors que le dieu VXOVINUS n'est attesté que par une seule inscription en territoire ligure et que les deux premières lettres VX, pourraient n'être qu'un rhabillage celtique.

\section{Jacques Dupoux $\uparrow$}

14 G. Barruor., Les peuples pré-romains du Sud-Est de la Gaule, $1^{\text {er }}$ suppl. à la Revue Archéologique de la Narbonnaise, Paris, 1959, p. 203-206; Pline l'Ancien, H.N., III, 34.

N.B. - Les illustrations sont dues à la collaboration de : R. Albert (fig. 3); L. Brissaud (fig. 4); R. Gesell (fig. $1,6,8,9,10 \mathrm{a}$ et b); Y. Marsoin (fig. 7 et $10 \mathrm{c}$ ); P. Trotignon (fig. 2). 